\title{
Tabularia
}

\section{Le ReTeHNor : un répertoire numérique de l'hagiographie latine de Normandie}

The ReTeHNor: A digital repertoire of Latin Hagiography of Normandy Il ReTeHNor: Un repertorio digitale di agiografia latina di Normandia

\section{Laura Vangone}

\section{OpenEdition}

\section{Journals}

Édition électronique

URL : http://journals.openedition.org/tabularia/3167

DOI : 10.4000/tabularia.3167

ISSN : 1630-7364

Éditeur :

CRAHAM - Centre Michel de Boüard, Presses universitaires de Caen

\section{Référence électronique}

Laura Vangone, "Le ReTeHNor : un répertoire numérique de l'hagiographie latine de Normandie »,

Tabularia [En ligne], Les sources des mondes normands à l'heure du numérique, mis en ligne le 23 novembre 2018, consulté le 19 avril 2019. URL : http://journals.openedition.org/tabularia/3167 ; DOI : 10.4000/tabularia.3167 



\title{
Le ReTeHNor: un répertoire numérique de l'hagiographie latine de Normandie
}

\author{
The ReTeHNor: A digital repertoire \\ of Latin Hagiography of Normandy
}

\section{Il ReTeHNor: Un repertorio digitale di agiografia latina di Normandia}

\author{
LaUra VANGONE \\ Craham-UMR 6273, Université de Caen Normandie \\ laura.vangone@unicaen.fr
}

Résumé:

Les humanités numériques accompagnent de plus en plus le travail des chercheurs en leur permettant de mieux mener à terme leurs recherches et de mieux en exploiter les résultats. Ces technologies ont ainsi favorisé la naissance de nombreuses bases de données en ligne. Le ReTeHNor (Repertorium Textuum Hagiographicorum in Normannia conscriptorum), rassemblant les textes hagiographiques en latin écrits sur le territoire du duché de Normandie pendant la période ducale (911-1204), est donc l'un de ces répertoires numériques. Basé sur le modèle de la $B H L$, il rassemble des fiches décrivant au niveau historico-littéraire ces textes hagiographiques et permettant d'avoir, grâce à différents modes d'interrogation, une vision critique et statistique de l'hagiographie de Normandie: combien de foyers hagiographiques? Combien d'hagiographes? Quels modèles de sainteté? Quelles typologies d'écrits hagiographiques? Etc.

Mots-clés: hagiographie, outil numérique, duché de Normandie, humanités numériques, base de données, BHL, bibliothèque numérique, répertoire

\begin{abstract}
:
Researchers' work is more and more accompanied by the practice of digital humanities which allow them to better accomplish their research and better exploit the results. Thus, these technologies have stimulated the emergence of many databases online. So, the ReTeHNor (Repertorium Textuum Hagiographicorum in Normannia conscriptorum), which collect the Latin hagiographical texts written in the area of the duchy of Normandy during the ducal period (911-1204), is one of these digital repertoire. Based on the BHL model, it collects files describing these hagiographical texts from a historical and a literary point of view and enable to have, thanks to different modes of inquiry, a critical and statistic view of the hagiography of Normandy: how many centers of hagiographic production? How many hagiographers? Which models of sanctity? Which types of hagiographic writing? Etc.
\end{abstract}

Keywords: hagiography, digital humanities, duchy of Normandy, database, BHL, digital library, repertoire

Tabularia «Études», "Les sources des mondes normands...", 2018, p. 1-17, 23 novembre 2018

URL: http://journals.openedition.org/tabularia/3167 | DOI: 10.4000/tabularia.3167 
Riassunto:

L'informatica umanistica accompagna in misura sempre maggiore il lavoro dei ricercatori consentendo loro di meglio portare a termine le loro ricerche e di meglio impiegarne i risultati. Queste tecnologie hanno cosi favorito la nascita di numerose basi di dati. Il ReTeHNor (Repertorium Textuum Hagiographicorum in Normannia conscriptorum), che raccoglie $i$ testi agiografici in latino scritti sul territorio del ducato di Normandia durante il periodo ducale (911-1204), è quindi uno di questi repertori digitali: basato sul modello della BHL, esso raccoglie un insieme di schede che descrivono accuratamente da un punto di vista storicoletterario questi testi agiografici e che consente di ottenere una visione critica e statistica dell'agiografia di Normandia: quanti centri di produzione agiografica? Quanti agiografi? Quali modelli di santità? Quali tipologie di scritti agiografici? Etc.

Parole chiave: agiografia, corpus, ducato di Normandia, informatica umanistica, base dati, $B H L$, biblioteca digitale, repertorio

Cet article est la version remaniée d'une communication prononcée lors de la journée d'étude «Les sources des mondes normands à l'heure du numérique» organisée par le Craham à l'Université de Caen Normandie le 16 février 2018. Je remercie les organisateurs de m'avoir invitée à présenter mes réflexions dans ce cadre.

\section{Introduction}

L'établissement de la base de données qui va être présentée dans cet article illustre le rôle créatif que les humanités numériques ont joué ces dernières années, en permettant la réalisation de projets toujours nouveaux et différents. Cependant, comme ces projets suscitent souvent la pensée que chaque solution imaginée dans le domaine des humanités numériques donne naissance à de nouveaux problèmes ${ }^{1}$, nous voudrions montrer pourquoi et comment nous avons été amenée à créer un répertoire numérique de fiches concernant des textes hagiographiques et quelle est son utilisation ${ }^{2}$. Ce projet fait partie d'un travail

1. BURDICK et al., 2012, p. 5 .

2. Plus particulièrement, dans le cadre de la recherche, le développement des techniques numériques semble avoir encouragé la création de corpora à des fins d'acquisition, de traitement et de restitution des données, et cela surtout dans le domaine des SHS, où le corpus est considéré comme l'objet de critères heuristiques de différents genres (chronologique, historique, littéraire, géographique), à la différence de ce qui se passe dans le cadre des études de linguistique où la réflexion est un peu plus avancée. Voir, par exemple, la revue en ligne Corpus [http://journals. openedition.org/corpus/] ou TogNINI-BonELLI, 2001. En fait, les problèmes inhérents à la définition de corpus sont relativement bien connus et débattus. Il n'existe pas de fixation conceptuelle unanime: l'existence du corpus est conçue de manière relationnelle et dépend du point de vue qu'on adopte afin de l'étudier. La diversité des objectifs et des résultats attendus de la constitution et de l'exploitation de corpora, dans les domaines de l'histoire ou de la littérature, détermine une diversité d'approches qui rend difficile l'énoncé d'une règle générale. Il est clair qu'un texte est un objet moins facile à évaluer qu'une inscription ou une monnaie, mais, quoi qu'il en soit, chaque corpus constitué pour décrire et/ou étudier un objet (qu'il s'agisse d'un texte ou d'un objet matériel) est nécessairement basé sur des critères subjectifs définis par son concepteur. Dans ce 
de thèse, en cours de réalisation au Craham (UMR 6273 CNRS/ Université de Caen Normandie), dont l'aspect numérique - l'encodage de l'ensemble des textes constituant le répertoire - bien qu'il soit au cour de la recherche, est accompagné d'un commentaire et d'une analyse des données tirées du répertoire. Je réalise donc un répertoire, le ReTeHNor (Repertorium Textuum Hagiographicorum in Normannia conscriptorum), qui propose une analyse de tous les textes hagiographiques écrits sur le territoire du duché de Normandie entre 911 et 1204. Il est constitué de 129 fiches - une pour chaque unité textuelle - et il est destiné à un public varié. Il s'agit d'une base de données «documentaire», dépourvue du texte hagiographique lui-même, mais donnant des nombreuses informations de nature historico-littéraire. On verra que la délimitation de cette base pose des problèmes qui m'ont amenée à créer aussi un Appendice comprenant les textes hagiographiques qui échappent à ces paramètres.

\section{L'état des études hagiographiques en Normandie}

Avant de présenter le ReTeHNor, il convient de rappeler brièvement que les recherches sur les textes hagiographiques ont connu un développement à partir des années $1960^{3}$ et sont désormais très vivantes en Normandie. Vers la fin du $\mathrm{XIX}^{e}$ et le début du XX ${ }^{e}$ siècle déjà, plusieurs œuvres érudites ont été consacrées aux saints vénérés dans la province normande 4 . Entre 1970 et 1990, Jean Fournée et Lucien Musset ont contribué au renouvellement de l'historiographie hagiographique sur la Normandie 5 . En 1975, Jean-Claude Richard soutenait sa thèse sur les Miracula écrits en Normandie, dont il a fourni, pour certains, la première édition ${ }^{6}$. En 1996, se tenait à Cerisy-la-Salle le colloque Les saints

sens, j'ai établi un corpus en faisant des choix guidés par la volonté d'obtenir certaines réponses et la structure du répertoire reste toujours modifiable en cours de recherche et selon ses besoins. Voir également infra, Un répertoire évolutif et ouvert: l'Appendice.

3. Dès lors (en 1967 est parue l'édition de la Vita Martini par J. Fontaine), les médiévistes ont commencé à s'intéresser d'une manière neuve aux modèles de sainteté et à l'étude des textes hagiographiques, y compris dans le domaine des sciences sociales et de l'histoire des mentalités, de par l'influence que le culte de saints et la littérature relative ont eue dans la société médiévale. Ainsi, C. Leonardi a pu écrire que: «La coincidenza tra coscienza ermeneutica e nuove consapevolezze storiografiche ha dunque portato a superare l'aspetto apologetico ancora presente nella tradizione bollandista, e a inserire a pieno titolo l'agiografia, dai margini in cui era rimasta per secoli, nella ricerca scientifica", dans Agiografia, in Medioevo latino, vol. 1, t. II, p. 430. L'étude des reliques a dû attendre le début des années 1970 avec l'œuvre de P. Geary sur les furta sacra (GeArY, 1993) et de M. Heinzelmann sur les translationes (HeinzelmanN, 1979), mais déjà P. Brown (Brown, 1984), en avait bien souligné les enjeux. Sur le rôle des reliques au Moyen Âge, voir également Bozóky, 2006.

4. À titre indicatif, voir Blin, 1873; Delamare, 1919; Mesnel, 1912-1918; PigeOn, 1891; Tougard, 1872; Tougard, 1884; Tougard, 1899; Tougard, 189o. Il faut rappeler aussi le travail de BAEDORF, 1913.

5. Encore à titre indicatif dans une bibliographie très vaste, voir FournéE, 1973; MUSSET, 1961-1962; Musset, 1970; Musset, 1975.

6. RICHARD, 1975. 
dans la Normandie médiévale ${ }^{7}$, alors même que Felice Lifshitz avait déjà fait son étude du dossier de saint Romain ${ }^{8}$. En 2009, P. Bouet et O. Desbordes ont publié l'édition des textes latins concernant l'histoire du Mont Saint-Michel comprenant des Miracula ainsi qu'une translatio et la célèbre Introductio monachorum $^{9}$. À l'heure actuelle, les investigations se tournent vers les rapports entre le culte des saints et le pouvoir, comme le montre l'ouvrage de S. Kahn Herrick pour la Normandie ${ }^{10}$, ainsi que la thèse de Lucile Trân-Duc, soutenue en 2015 à l'université de Caen Normandie: focalisée sur l'hagiographie du seul diocèse de Rouen, elle analyse les rapports des sources hagiographiques avec le pouvoir ducal et entre les différents établissements dans le cadre de la reconstitution de la vie religieuse au lendemain des invasions scandinaves ${ }^{11}$.

\section{Un répertoire clos: le ReTeHNor}

C'est donc dans ce même domaine que s'inscrit ma thèse de doctorat qui vise à recenser la production hagiographique de la Normandie ducale puis à en donner une analyse littéraire et historique. Le ReTeHNor est constitué de fiches synthétiques et riches de données historiques et philologiques pour chaque texte hagiographique latin écrit sur le territoire de la Normandie pendant la période ducale (911-1204) $)^{12}$, dont on précise aussi le «genre» (Vitae, Passiones,

7. Les saints..., 2000 .

8. Pour sa thèse de doctorat (The Dossier of Romanus of Rouen: The Political Uses of Hagiographical Textes, thèse de doctorat inédite, Columbia University, 1988) puis remaniée et publiée en 1995 (The Norman Conquest of Pious Neustria: Historiographic Discourse and Saintly Relics, 684-109o. Toronto, Pontifical Institute of Mediaeval Studies, 1995).

9. Bouet, Desbordes, 2009.

10. KAHN HERrick, 2007.

11. TrÂN-DUC, 2015 .

12. Précisons que nous ne tenons pas compte de la provenance historique l'origine historique des saints: le répertoire contient des textes qui honorent les apôtres, la Vierge, des saints anglais, italiens, flamands, l'archange Michel... Le choix des textes qu'on insère dans le ReTeHNor suit un critère géographique qui, avec les critères littéraire et chronologique, guide la recherche: un hagiographe normand écrivant en Normandie sur un saint non normand ou pour un établissement situé hors de Normandie figure dans le ReTeHNor au même titre qu'un hagiographe non normand écrivant en Normandie sur des saints normands et/ou non normands (le cas le plus symptomatique est celui de Baudri de Bourgueil). La raison en est qu'on s'intéresse au travail des scriptoria, aux transferts culturels, aux sources qui se trouveraient trouvait en Normandie. Pour ce qui concerne les bornes chronologiques, le discours n'est plus simple qu'en apparence: sachant que le terminus ante quem de 1500 utilisé par les Bollandistes pour leur BHL n'avait pas historiquement raison d'être, et considérant que l'objectif de la thèse n'est pas seulement de faire un inventaire ou d'avoir une approche littéraire mais aussi historique, les années entre 911 et 1204, c'est-à-dire entre la naissance formelle de la Normandie et son annexion au royaume de France par Philippe Auguste, suffisent parce qu'elles offrent en elles-mêmes les conditions réelles pour l'étude des transformations historiques (qui jouent un rôle important dans l'écriture hagiographique). Elles permettent aussi d'étudier des formes de discours tenus sur les Normands, vus d'abord comme des pillards puis présentés comme des participants très actifs des croisades, et le rôle joué par l'hagiographie - et le culte des saints - dans l'organisation politique, sociale et territoriale de cette nouvelle et très singulière entité que fut la Normandie: Cassandra Potts parle de "one religious unit under the same body of saints». Cf. Роттs, 1995, p. 17. 
Translationes, Miracula, etc.). Le ReTeHNor, en se donnant comme critères ontologiques des données chronologiques, géographiques, historiques ainsi que littéraires (textes hagiographiques, en latin ${ }^{13}$ ), se fixe un double but ou plutôt un but unique (quelles sont les caractéristiques de l'hagiographie normande latine à l'époque ducale) mais répondant à deux questions de nature complémentaire: 1) la première est d'ordre littéraire (qui sont les hagiographes, combien de textes ont-ils écrits, où et comment?) 2) la deuxième est d'ordre historique (et répond essentiellement à la question: «pourquoi a-t-on écrit?»). Le ReTeHNor est alors un outil pour mes recherches en thèse, me permettant de tirer des données qui sont ensuite analysées de façon discursive de ce double point de vue.

Le ReTeHNor s'inspire principalement pour sa structure de la Bibliotheca Hagiographica latina antiquae et mediae aetatis $(B H L)^{14}$ associé à la Bibliotheca Hagiographica Latina manuscripta ${ }^{15}$ (BHLms) en ligne ${ }^{16}$. La réalisation de la $B H L$ fut un exploit remarquable pour son époque ${ }^{17}$ et représente la base de départ du travail: ce répertoire alphanumérique a permis de distinguer dans un premier temps environ 13600 items dont la plupart étaient anonymes et munis seulement d'un identifiant «de genre» (par exemple, Vita, Miracula, Passio, etc.), qui rendait les textes indifférenciables selon les critères historico-littéraires.

13. Une histoire générale de l'hagiographie n'existe pas encore et «même pas un ensemble d'histoires particulières, synchroniques ou diachroniques, qui pourraient, mises bout à bout, donner une vue d'ensemble de la matière». Cette observation, accompagnée d'une explication du phénomène, ouvre, à travers les mots de G. Philippart, le recueil d'Hagiographies. Histoire internationale de la littérature hagiographique latine et vernaculaire en Occident des origines à 1550, Turnhout, Brepols, 1994, (Corpus Christianorum, Hagiographies, 1), p. 10. C'est pour cette raison que F. Dolbeau pouvait conclure, dans son bilan des études hagiographiques en France, que «l'idéal serait d'aborder les textes hagiographiques d'une part en séries cohérentes du point de vue de leur lieu de production ou à défaut de leur origine géographique». Cf. Dolbeau, 1999, p. 66. C’est donc à ce critère que répond, dans les grandes lignes, l'idée de rassembler les textes hagiographiques du duché de Normandie.

14. Bibliotheca Hagiographica latina antiquae et mediae aetatis, éd. Société des Bollandistes, Bruxelles, Société des Bollandistes, 1898-1899, t. 1 (A-I), t. 2 (K-Z) (rééd. anastatique, Bruxelles, 1992); Supplementi editio altera auctior, Bruxelles, Société des Bollandistes (Subsidia Hagiographica 12), 1911 (la première édition de ce supplément était parue à la fin du dernier fascicule de la $B H L$, p. 1305-1387 mais, devenu inutile, il a ensuite été supprimé dans les impressions anastatiques successives de la $B H L)$; Bibliotheca hagiographica latina antiquae et mediae aetatis. Novum Supplementum, éd. H. Fros (Subsidia Hagiographica 70), Bruxelles, 1986.

15. Cette base de données a le mérite de relier les sources de la $B H L$ avec celles des catalogues des manuscrits hagiographiques publiés dans la série des Subsidia Hagiographica, des Analecta Bollandiana et d'autres revues internationales.

16. Le modèle de la $B H L$ a déjà inspiré d'autres travaux de mise à jour et de répertoriage. Voir, par exemple, Dolbeau, 1994 et Verrando, 1995.

17. Elle a été le résultat d'environ 350 années de travail de recherche ininterrompu et a eu pour objectif de recueillir tous les textes hagiographiques en latin écrits avant 1500, en excluant les inscriptions, les hymnes et, plus généralement, tous les textes non narratifs. Pourtant, son immédiateté ne doit pas nous faire oublier qu'elle est le fruit de siècles d'exceptionnelle érudition: les Acta Sanctorum (AASS) furent initiés par le belge Jean Bolland (1596-1665) et poursuivis par d'autres pères jésuites. Cf. Acta Sanctorum, Anvers-Bruxelles, Société des Bollandistes, 1643-1940, 68 vol. (rééd. Turnhout, Brepols, 1966-1971); $2^{\mathrm{e}}$ éd., Venise, 1734-1770, 43 vol.; $3^{\mathrm{e} e ́ d ., ~ P a r i s-R o m e, ~}$ V. Palmé, $1863-1887,60$ vol. 
L'objectif du ReTeHNor est donc d'intégrer les données évoquées ci-dessus et, lorsque cela est possible, de les organiser pour établir un véritable status quaestionis ouvert et configuré de façon critique. La diversité et le nombre accru des champs, par rapport à la $B H L$ et à la $B H L m s$, m'ont amenée à approfondir et à rendre explicites de nombreux aspects du texte hagiographique qu'il devient dès lors possible d'étudier. Plusieurs savants ont déjà travaillé dans ce sens, parmi lesquels notamment les auteurs des SHG, les Sources hagiographiques de la Gaule avant l'an Mil, sous la direction de $\mathrm{M}$. Heinzelmann ${ }^{18}$. Pour la réalisation de cette publication, les chercheurs se sont servi de la $B H L$, mais aussi de la série de Catalogues de manuscrits hagiographiques latins publiés dans les Analecta Bollandiana et les Subsidia Hagiographica ainsi que d'études particulières menées sur un texte, un auteur, un saint ou un diocèse pendant ces dernières années ${ }^{19}$; quand aucune étude ou analyse n'avait été faite sur un texte ou un dossier, j'ai mené ma propre investigation, afin de pouvoir établir l'existence de liens avec l'hagiographie de la période ${ }^{20}$.

La réalisation du ReTeHNor donc n'est pas seulement une question de mise à jour matérielle ou de "support» (papier versus informatique); il s'agit aussi d'un travail critique qui a abouti à la création de la $B H N$, un acronyme parlant (Bibliotheca Hagiographica Normanniae) ${ }^{21}$ : chaque texte faisant l'objet d'une fiche dans le ReTeHNor (territoire de Normandie, 911-1204) a reçu un numéro $B H N$. Pour ce faire, certains items $B H L$ ont été fusionnés (par exemple, là où la $B H L$ sépare le prologue du texte auquel il appartient en donnant deux identifiants différents); d'autres ont été subdivisés (dans le cas de réécritures ${ }^{22}$ ); d'autres encore ont été ajoutés quand, pour différentes raisons, ils n'ont pas de numéro $B H L^{23}$ (BHL vacat) ${ }^{24}$. La BHN représente donc le principe conceptuel,

18. Voir Dolbeau et al., 1987.

19. Pour n'en citer que quelques-uns: Dolbeau, 1986; M. Hinkner, 2006; Le Hü̈rou, 2013; Le Мано, 2006.

20. Comme dans le cas du dossier de saint Taurin où, bien que la Vita ait été étudiée par S. Kahn Herrick (KAHN HerRICK 2007, p. 51-73), les deux Vitae (BHN 107/108-BHL 7990/7991) et les trois Inventiones et miracula (BHN XCV/109/XCVI - BHL 7992/7993/7994), correspondent toutes à des versions différentes qui ont été ramenées au lieu de leur rédaction; ou comme dans le cas de la Passio Maximi et Venerandi (BHN 73/BHL 5848): j'ai découvert qu'il s'agit du plagiat presque intégral d'une autre Passio, celle des saints Savin et Cyprien ( $B H L$ 7447), très probablement réalisé au moment où Acquigny (dép. Eure, arr. Évreux) devint un prieuré fontevriste pendant la première moitié du XII ${ }^{e}$ siècle. On peut remarquer comment on a également utilisé un identifiant alternatif pour les textes de l'Appendice: au lieu des chiffres arabes, il y a les chiffres romains.

21. Voir Dalarun et al., 1994.

22. Sur le phénomène de la réécriture, voir Goullet, 2005. Si on regarde le specimen de la fiche infra, on verra que pour chaque numéro $B H N$ est donnée la référence $B H L$.

23. Il peut s'agir par exemple de textes dont on a découvert des réécritures ou des versions plus anciennes grâce à une étude plus attentive des manuscrits comme dans le cas des dossiers de saint Évroult (Chibnall 1972) ou de saint Godard ou encore de Haimon, moine de Savigny (WALKER 2004) ou de textes qui n’ont simplement pas été répertoriés par la $B H L$ (comme certains des miracula édités par J.-C. Richard ou la Vita Ceronnae publiée par F. Dolbeau).

24. On a été amené à ces choix par le fait que les Bollandistes, dans leur édition des AASS, n'indiquent pas les manuscrits dont ils se servent ou bien ils découpent les textes d'une façon qui ne reflète pas la réalité matérielle des manuscrits, et cela aussi quand ils font référence à des éditions réalisées 
formel, on pourrait dire le «template», modèle de présentation des données, signalant en même temps le modèle fondamental (la $B H L$ ) et fournissant un identifiant à chaque texte (comparable et substituable à celui de la $B H L$ ), alors que le ReTeHNor offre une analyse concrète du texte consultable soit au format papier soit au format informatique.

Il existe déjà plusieurs bases de données à usage hagiographique plus ou moins exclusif ${ }^{25}$, bases textuelles et bases documentaires ${ }^{26}$. C'est la possibilité de numériser les données qui rend utile la réalisation de ce répertoire, car la structure de la base de données permet de multiplier les modes d'interrogation des données, mais aussi autorise la mise à jour progressive au fur et à mesure de l'avancée des recherches, ce que le papier n'est pas en mesure d'offrir. Le ReTeHNor, comme on l'a déjà dit, se présente donc comme un dossier «documentaire». Lorsqu'il sera disponible en ligne - au plus tard dans les six prochains mois - il sera possible de trier les textes chronologiquement, de faire des recherches par auteur, par lieu de production, par modèle de sainteté, par commanditaire, par typologie hagiographique.

par d'autres. En somme, dans la plupart des cas, la logique de la $B H L$ ne correspond pas à celle du scribe, comme l'a bien dit G. Philippart en écrivant que la $B H L$ a souvent répertorié «non des œuvres médiévales distinctes, mais des éditions modernes distinctes d'œuvres médiévales" (Cf. Philippart, 1992, p. 24). Qui plus est, les Bollandistes font généralement des choix éditoriaux qu'ils ne communiquent pas aux lecteurs. Pour cette raison, par exemple, on a éliminé les titres utilisés par la BHL (Vita, Passio, Translatio et Miracula, etc., souvent accompagnés par une indication géographique, le nom de l'auteur ou la datation quand où ils sont connus), car il n'est quasiment jamais possible de savoir s'ils viennent du manuscrit lui-même ou s'ils ont été ajoutés ultérieurement par un copiste ou un éditeur. En fait, M. Goullet a souligné la pluralité des «genres» employés par la $B H L$ pour désigner les textes hagiographiques. Ils sont au nombre de 76. Elle ajoute: "Ces "genres" ne sont pas du tout homogènes, puisqu'à côté de catégories bien connues comme Miraculum ou Passio on y distingue des titres français - assez énigmatiques quand ils s'appliquent à des textes - comme "reliques", "lettre ou chiffre", et des notions qui relèvent davantage de l'unité codicologique (libellus) ou éditoriale (liber, prologus, epilogus) que du genre littéraire». Cf. Goullet 2001, p. 141.

25. En premier lieu, la BHL manuscripta (http://bhlms.fltr.ucl.ac.be/) ou le travail entamé par G. Philippart à l'Université de Namur (http://www.unamur.be/philo_lettres/histoire/h221m.htm) qui n'est pas exhaustif. Par ailleurs, ce dernier travail est étroitement lié à la BHLms qui dérive d’une autre base de données, «Légendiers», établie sous la direction de G. Philippart et réalisée en collaboration avec les Bollandistes. Cf. De VRIendt, Trigalet 1997, p. 5-16.

26. Voir tout d'abord l'AASS Database, spécifiquement hagiographique. Citons aussi le travail en cours mené dans le cadre du Projet CBMA - Corpus Burgundiae Medii Aevi. Site du projet Corpus de la Bourgogne du Moyen Âge, consultable en ligne: http://www.cbma-project.eu. Il s'agit d'une plate-forme documentaire de la production diplomatique de Bourgogne qui inclut désormais progressivement d'autres types de sources, parmi lesquelles figurent les sources hagiographiques, pour arriver à la constitution d'un corpus hétérogène et interrogeable au moyen des autres outils mis à disposition par les humanités numériques comme le SIG ou la lemmatisation. Cf. MAGNANI 2017, p. 59-65. Il faut mentionner aussi les autres grandes bases de données textuelles en latin, qui donnent à lire, parmi d'autres sources, des textes hagiographiques, comme LLT-O (Library of Latin Texts - online, http://www.brepols.net/Pages/BrowseBySeries. aspx?TreeSeries=LLT-O), $d M G H$ (Monumenta Germaniae Historica digital, http://www.dmgh. de/), ALIM (Archivio della Latinità Italiana del Medioevo, http://www.alim.dfll.univr.it/), Corpus Corporum (http://mlat.uzh.ch/MLS/index.php?lang=o), Musisque Deoque (http://www.mqdq.it/ public/), PHI (Packard Humanities Institute) Latin Texts, (http://latin.packhum.org/). 
Chaque fiche est structurée selon l'exemple suivant:

\author{
AGNES V. M. ROMAE \\ BHN 2 \\ Référence BHL 164b \\ AGNES V. M. ROMAE \\ Dates du saint : \\ 290/293-305 \\ Modèle de sainteté: \\ Martyr \\ Typologie hagiographique: \\ Passio \\ Incipit: \\ "Virgae regalis de germine flos specialis...» \\ Explicit: \\ «...Error damnatur, rex regum magnificatur» \\ Forme: \\ En vers \\ Auteur: \\ Monachus Rotomagensis quidam \\ Commanditaire: \\ Nicolas, abbé de Saint-Ouen (?) \\ Lieu de rédaction: \\ Abbaye Saint-Ouen de Rouen \\ Datation du texte:
}

Deuxième moitié du XI ${ }^{\mathrm{e}}$ siècle

Éditions:

Dolbeau F., Deux poèmes inédits, extraits du «Livre noir» de Saint-Ouen de Rouen, dans Sanctorum societas. Récits latins de sainteté (III $-\mathrm{XII}{ }^{\mathrm{e}}$ siècle), Bruxelles, Société des Bollandistes, 2005 (Subsidia hagiographica, 85), t. II, p. 766-773.

Manuscrits :

Rouen, BM, Y.41 (1406), fol. 69v; 80-83, fin du XIe-début du XII e siècle

Résumé critique:

Ce texte est une passion versifiée de la martyre romaine Agnès en 202 hexamètres dactyliques. Il s'agit d'une réécriture de la Passio pseudo-ambrosienne en prose (BHL 156), elle-même copiée sur les feuillets précédents du Livre Noir de l'abbaye de Rouen (Rouen, BM, Y.41, fol. 61v-69v); l'abbaye possède le chef de la sainte. F. Dolbeau, le premier éditeur du texte, en a rectifié l'erreur attributive due à Dom Pommeraye et suivie par l'Histoire littéraire. Dom Pommeraye avait créé le fantôme d'un moine rouennais nommé Ambroise d'après la salutatio de la Passio en prose «Servuus Christi Ambrosius virginibus sacris» en lui attribuant les deux pièces sur sainte Agnès et en faisant un opus geminum produit du même auteur. En fait, Ambroise était l'évêque de Milan (374-397) et Dolbeau démasque cette grossière erreur et nous donne une très bonne édition critique avec analyse stylistique (qui met en exergue les liens de ce texte-ci avec BHL 6083, BHL 7310/7311 et BHL 6127d sans pourtant arriver à affirmer une même paternité) et métrique, qui révèle la formation de haut niveau de l'écrivain en ce domaine. Une copie de ce texte subsiste dans un manuscrit des Bollandistes (Bruxelles, Bibl. Boll., 104bis, fol. 125-128v). 
Donc, par rapport aux données qu'on pouvait tirer de l'étude jointe de la BHL et de la BHLms, ce répertoire a le mérite de fournir un plus grand nombre de données synthétiques - le lieu de rédaction (atelier régulier ou séculier), le nom de l'auteur en latin et au nominatif (ou au moins sa provenance, là où la BHL donne seulement les noms des auteurs connus), l'aspect formel du texte (prose, verse, prosimètre), le ou les commanditaires, une datation ou une hypothèse de datation. Ne pas dater ou ne pas donner une datation approximative d'un texte hagiographique empêche de le contextualiser davantage. Pour autant, définir l'époque où un texte a été rédigé n'est pas toujours une tâche facile en hagiographie; pour les textes les plus difficiles à dater précisément, nous avons recours au terminus ante quem qui est de manière générale fourni par la datation du témoin manuscrit le plus ancien - du moins quand celle-ci est établie selon des critères paléographiques et codicologiques. Ce terminus ante quem peut être contrebalancé par un terminus post quem représenté, faute d'autres renseignements, par l'époque où l'on suppose que le saint a vécu ou, là où ils existent, par d'autres écrits concernant le même saint et dont on est sûr de l'antériorité. Les témoins manuscrits sont listés du plus ancien au plus récent et les données les concernant sont présentées de gauche à droite dans l'ordre suivant: ville où le manuscrit est conservé, sigle de la bibliothèque, cote, numéro des folia, datation (la datation est en chiffres romains pour indiquer le siècle, à moins qu'on ne connaisse une datation ad annum indiquée en chiffres arabes, qui peut être suivie par la mention $c a$. [circa] pour signifier un laps de temps approximatif). Là où deux manuscrits appartiennent de façon générique à la même période (normalement, le même siècle), on a suivi le critère alphabétique pour les lister. La liste des témoins manuscrits est précédée par une liste des éditions des plus anciennes aux plus récentes, qui peuvent être accompagnées ou supplantées - dans le cas de textes inédits - par des indications bibliographiques contenant des excerpta du même texte (la BHL fournissait déjà une liste des œuvres imprimées suivie par celle des œuvres mentionnant excerpta et/ ou variae lectiones). Toutes ces données sont associées à une discussion critique des études, mises à jour, qui tracent l'histoire du texte dans ses différents aspects historiques et philologiques. Bien évidemment, la structure de la fiche a été enrichie au fur et à mesure de l'avancement du travail: au début de la thèse, elle ne contenait que l'incipit, l'explicit, l'auteur, la datation, les éditions, les manuscrits et le résumé critique. Tous les autres champs, ajoutés au cours de la recherche, ont précisément pour but de décrire plus en détail le texte afin d'en rendre compte de sa spécificité et d'en augmenter les champs d'exploitation numériques.

\section{Pourquoi une structure numérique?}

Une fois la version papier du ReTeHNor établie, j'ai pu envisager la transposition des fiches hagiographiques dans le ReTeHNor numérique grâce à l'aide du Pôle Document Numérique (PDN) de la MRSH de l'université de Caen Normandie ${ }^{27}$.

27. Je remercie grandement le Pôle Document numérique de la MRSH de Caen et notamment Marie Bisson et Subha-Sree Pasupathy pour leur aide précieuse dans le développement de cette interface. 
Après avoir stabilisé la structure des fiches dans un traitement de texte, nous avons choisi de les convertir au format XML-EAD: standard informatique utilisé pour réaliser des catalogues, des inventaires ou des répertoires de fonds d'archives, de manuscrits ou de collections. Pour faciliter le travail complémentaire sur ces fiches, un environnement de travail a été configuré à l'aide du logiciel XML Mind XML Editor. L'exposition et la consultation finale des fiches sont réalisées grâce à l'outil de publication et de diffusion Pleade (version 3.4).

La fiche convertie en XML-EAD est présentée infra.

L'avantage d'une structuration numérique est de plusieurs ordres. Elle offre tout d'abord la possibilité de constituer les dossiers hagiographiques les plus complets possible en utilisant des ressources à la fois anciennes et modernes. Le ReTeHNor réunit des données provenant de sources et structures ( $B H L$ papier - BHLms numérique) différentes, permettant de les rechercher et de les exploiter d'un seul coup, en éliminant ainsi le besoin d'un recours différencié aux sources. Un plus grand nombre de données est alors converti de façon plus souple dans un répertoire numérique, surtout parce qu'on peut y associer de cette façon la littérature secondaire et une bibliographie mise à jour, ce qui manque complètement dans la $B H L$. Ce travail est rendu possible aussi, parce que les outils numériques n'effacent pas les méthodes et les études déjà existantes, mais au contraire exploitent les connaissances acquises depuis longtemps et donc propres à une discipline. La structuration numérique associée à son exploitation sur le web offre aussi la possibilité d'ajouter des liens hypertextuels: de cette façon, les différents domaines d'application des humanités numériques peuvent se rencontrer et s'enrichir mutuellement. La multiplication de la numérisation des manuscrits hagiographiques peut, par exemple, étoffer la fiche et aider à avoir une approche plus critique envers les éditions anciennes - qui souvent ne sont pas des éditions critiques au sens vrai et moderne du terme. On pourra également, à l'avenir, élargir la base documentaire et réaliser une base textuelle de l'hagiographie de la Normandie: cela facilitera aussi la réalisation d'éditions savantes numériques qui, notamment dans le cas de l'hagiographie, permettent de dépasser les limites de l'édition traditionnelle et de rendre compte des nuances rédactionnelles les plus petites ${ }^{28}$. De plus, la base textuelle, notamment à travers l'analyse des espaces lexicographiques, peut apporter une grande aide dans les attributions d'auteur.

Naturellement, pour la constitution d'un répertoire, il faut choisir des données, les extraire, les délimiter, les transformer, les homogénéiser, les organiser, tant intellectuellement que matériellement pour faire d'un ensemble, un corpus. L'établissement d'un corpus exige alors la capacité critique de classer, de hiérarchiser et établir des liens entre les différentes parties dont il est formé. De ce point de vue, l'apport des humanités numériques permet de créer des connexions: le répertoire sera ainsi interrogeable sur la base, par exemple, de la recherche de l'incipit ou de l'explicit (une fonction qui est encore expérimentale

28. Voir Pierazzo, 2015. 


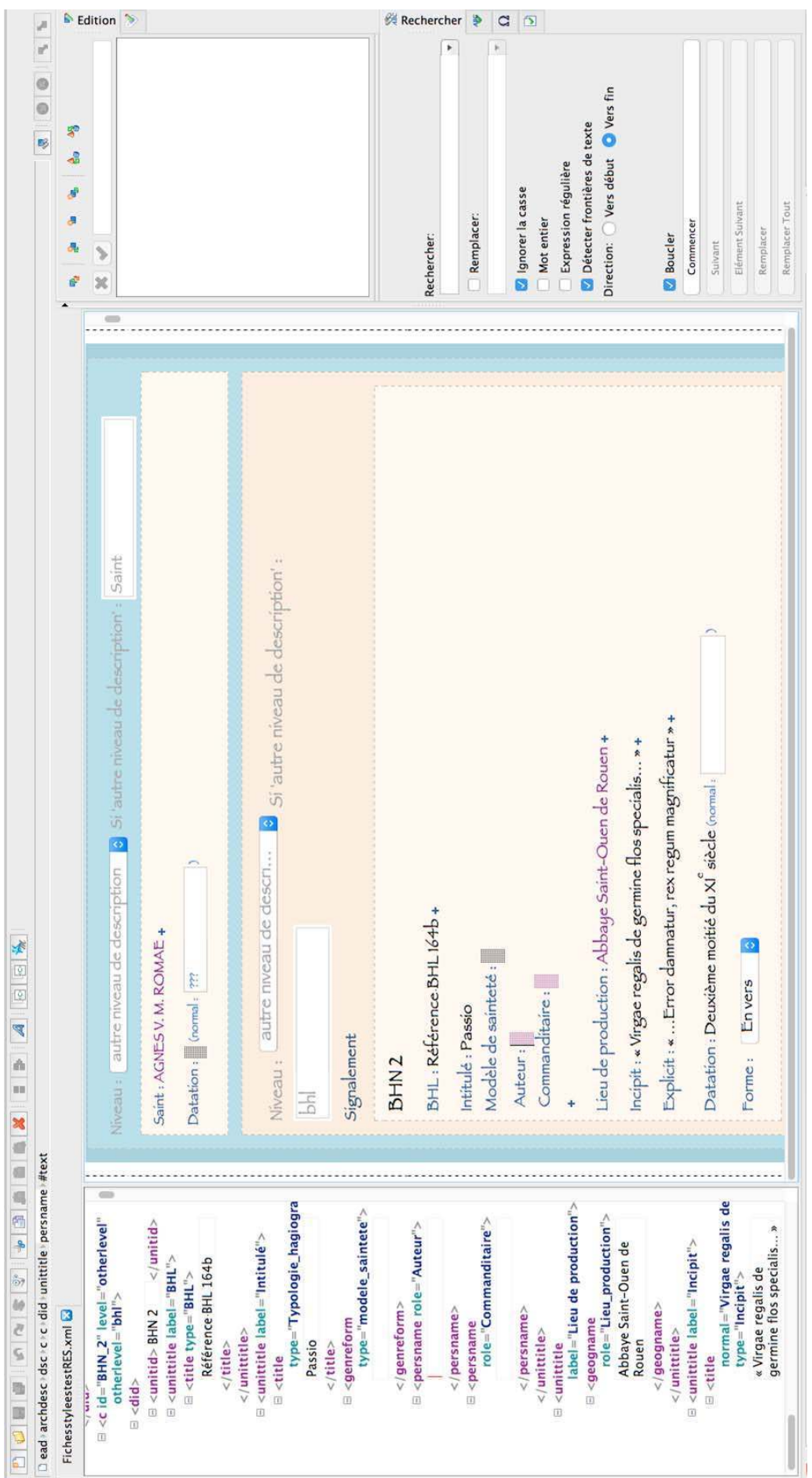

Tabularia «Études», «Les sources des mondes normands...», 2018, p. 1-17, 23 novembre 2018 
pour la $B H L m s^{29}$ ), de l'auteur, du lieu de rédaction, des commanditaires, encore de la datation, du modèle de sainteté... bref, il permettra de répondre à des questions qui, dans leur ensemble, sont encore sans réponse pour ce qui concerne l'hagiographie de Normandie et d'utiliser ces réponses comme point de départ pour se poser d'autres interrogations ${ }^{30}$. En outre, le répertoire donne la possibilité d'effacer les erreurs de datation que la numération $B H L$ peut causer (par exemple, à l'intérieur du dossier d'un saint, un numéro $B H L$ plus haut indique un texte plus ancien mais cela ne reflète pas toujours la réalité). Ainsi, les outils numériques multiplient les modes d'accès aux données et réduisent le problème de l'ordre de présentation imposé par l'organisation du support matériel. Last but not least, le répertoire numérique permet l'addition de nouveau matériel: la découverte d'un nouveau texte, d'une nouvelle attribution ou datation pourra vite être ajoutée au moyen d'une nouvelle fiche. En dernier lieu encore, un tel répertoire admet que, à travers des systèmes d'information géographique (SIG), on puisse envisager l'établissement d'une cartographie des productions écrites.

\section{Un répertoire évolutif et ouvert: l'Appendice}

Le ReTeHNor, comme on vient de le voir, voudrait proposer des fiches pour tous les textes hagiographiques écrits sur le territoire de la Normandie ducale entre 911 et 1204 en constituant ainsi un répertoire inédit, à la fois riche et synthétique. Dans le cas de l'hagiographie, on pourrait dire qu'on a affaire à des répertoires déjà constitués, où tous les textes concernant un même saint sont regroupés dans un dossier: ce dossier est un répertoire articulé sur le nom et le culte du saint. Comme on l'a déjà dit au début, d'une part, la définition des critères de validité des bornes heuristiques d'un répertoire - base méthodologique pour le développement des recherches -, et, d'autre part, l'évolution des humanités numériques ont amené les chercheurs à mieux définir le concept de corpus. Cécile Treffort a écrit que "Le corpus idéal se prête mal à une formalisation définitive, car son élaboration accompagne étroitement le processus intellectuel de la recherche. Obligeant à définir, à choisir et à éliminer, il se bat constamment contre l'intelligence historique consciente de la complexité, de la nuance, de la mutabilité des choses, des faits ou des signes ${ }^{31}$. En effet, si notre recherche porte

29. Les résultats de cette recherche ne sont pas fiables parce que seulement une partie des incipit ont été renseignés; il s'agit en tout cas, pour la BHLms, d'un travail en cours dont la dernière mise à jour remonte à 1998 .

30. Si un chercheur veut étudier un dossier hagiographique précis, il pourra par exemple utiliser le répertoire pour évaluer le status quaestionis et procéder à l'édition de textes inédits, mal ou très anciennement édités; il pourra aussi rechercher des données utiles à ses propres recherches dans tous les genres - historique, sociologique, littéraire, codicologique, etc. En fait, le ReTeHNor a aussi le mérite de donner d'une manière immédiate la mesure du traitement inégal auquel les textes hagiographiques, parfois concernant le même saint, ont été soumis, notamment à cause de l'exploitation arbitraire dont ils ont fait l'objet de la part des chercheurs, attentifs à ce qui regarde de près leurs propres intérêts et problématiques. D'autre part, l'Appendice présente de très nombreux textes de datation incertaine qui attendent une étude plus approfondie.

31. TRefFort 2014 
sur l'hagiographie de Normandie à partir de l'étude des textes hagiographiques latins écrits en Normandie (et non en Angleterre ou en Italie du Sud) entre 911 et 1204, elle a pourtant souvent et nécessairement besoin de faire référence à ce qui est en dehors de ses limites heuristiques. En laissant de côté les difficultés inhérentes à la recherche en elle-même (datations, pluralité d'attributions, difficulté d'identifier le scriptorium, etc.), qui ont amené à l'insertion dans le répertoire de textes de datation incertaine ${ }^{32}$, un autre problème s'est posé dans la phase de création. Il s'agit de tous ces textes qui ont partie liée avec le discours sur l'hagiographie de Normandie mais qui ne respectent pas les critères heuristiques fixés en début de recherche (voir notamment le cas où un texte normand est une réécriture d'un texte écrit ailleurs et/ou avant 911). Alors, afin de rendre le répertoire mieux exploitable par les chercheurs, le ReTeHNor sera accompagné d'un Appendice en cours de réalisation, qui sera soumis au même traitement numérique que le ReTeHNor. L'Appendice est de ce fait un autre repertorium contenant des fiches (environ 110) de tous les textes hagiographiques qui, pour des raisons méthodologiques, ont été exclus du cœur de la recherche: en d'autres termes, il contient les fiches des textes hagiographiques liés d'un point de vue historico-littéraire à l'hagiographie de Normandie d'époque ducale mais qui n'entrent pas dans les critères du ReTeHNor ${ }^{33}$. Les textes de l'Appendice, bien qu'ils ne soient pas l'objet premier de la recherche, figurent à divers titres dans les arguments de la thèse ${ }^{34}$. Ainsi, pour un saint, le dossier comprend aussi les textes qui n'ont pas été écrits en Normandie entre 911 et 1204 figurant dans l'Appendice $^{35}$. Néanmoins, pour ceux qui voudront aborder une recherche à

32. Si un texte est une réécriture d'un texte de l'époque carolingienne et que son plus ancien témoin manuscrit remonte au $\mathrm{XI}^{\mathrm{e}}$ siècle, là où il n'y a pas d'études critiques, il peut s'agir d'un texte écrit au $\mathrm{IX}^{\mathrm{e}}$, au $\mathrm{X}^{\mathrm{e}}$ comme au $\mathrm{XI}^{\mathrm{e}}$ siècle: la question pourra être approfondie par d'autres chercheurs.

33. Le ReTeHNor, dans sa cohérence, ne résout pas ce que D. Mayaffre - bien qu'il applique ses réflexions aux corpora textuels - appelle la «réflexivité du corpus», c'est-à-dire le fait que ses parties renvoient les unes aux autres en formant un «réseau sémantique» (cf. MAYAFFre 2002); bien que le concept de réflexivité appliqué à l'hagiographie puisse apparaître fascinant (souvent un texte est réécrit et donc il est le reflet d'un autre texte), il n'est pas applicable dans notre cas: pour avoir un corpus réflexif, tous les éléments devraient avoir égale dignité, ce qui n'est pas le cas, car les textes qui sont dans le ReTeNHor ne sont pas traités comme ceux qui sont dans l'Appendice. De plus, on serait confronté à la difficulté d'établir si l'utilisation d'un texte par un autre texte est faite directement ou par l'intermédiaire d'un autre hypotexte. Le concept de réflexivité du corpus a par exemple été utilisé par S. Fray qui a enrichi rétrospectivement le corpus de sa thèse de doctorat sur L'aristocratie läque au miroir des récits hagiographiques des pays d'Olt et de Dordogne $\left[X^{e}-X I^{e}\right.$ siècles], avec des éléments externes en prenant en compte des documents diplomatiques dont il a réévalué les rapports avec les textes hagiographiques. Cf. MAGNANI, 2017, p. 9-10.

34. «...N'a-t-on pas besoin d'éléments étrangers - disons pour l'instant de la manière la plus vaste et la plus problématique, de notre culture - pour le (scil. le corpus) comprendre? Et ainsi ne se trouve-t-on pas projeté en dehors des limites objectives du corpus dans un tout-subjectif sans garde-fou scientifique ni garantie méthodologique? [...]. Elles (scil. les ressources extérieures) seront donc de même nature que le corpus, entraînant une confusion et une discrimination difficilement justifiable entre les textes du corpus traités scientifiquement et ceux de l'intertexte mobilisés sans traitement préalable». MAYAFFrE 2002, p. 57-58.

35. C'est par exemple le cas du dossier de saint Hugues, évêque de Rouen, dont le texte $B H N$ 56/ $B H L 4033$ est le seul du dossier rentrant dans le ReTeHNor en tant que réécriture due à Baudri de Borgueil et réalisée très probablement à Jumièges entre 1119 et 1127 (LE HUËroU, 2013, p. 163-189). 
partir de bases différentes des nôtres, le ReTeHNor permettra une recherche par dossier dans laquelle la distinction entre Répertoire et Appendice disparaitra. L'Appendice contient aussi les sources relatives à l'hagiographie carolingienne de Neustrie, mais pas les textes qui ont été hypotextes d'hagiographies écrites en Normandie (par exemple, dans le cas où une hagiographie normande réécrit un texte totalement étranger à nos critères) ${ }^{36}$.

La création d'un répertoire se révèle aussi fondamentale que problématique: elle est guidée par un esprit critique mais aussi pragmatique qui pousse à l'enrichissement du corpus avec des éléments externes. La possibilité d'ajouter du nouveau matériel représente une sorte d'arme à double tranchant et l'aspect le plus controversé de la recherche. Avec la création de l'Appendice, une question se pose: quand arrête-t-on d'y ajouter des fiches? Et surtout, l'établissement d'un Appendice ne fait-il pas disparaitre le choix documentaire premier? La réponse à cette question est, au moins pour le moment, négative, dans la mesure où les textes qui font l'objet de la thèse de doctorat - qui se déroule dans un temps limité - sont seulement ceux contenus dans le ReTeHNor: il s'ensuit que le ReTeHNor est le véritable objet de la recherche, tandis que l'Appendice est un moyen, fonctionnel à l'objet, car il contient des textes qui peuvent aider à mieux saisir certaines relations ou certains aspects des textes du ReTeHNor.

\section{Conclusions}

En conclusion, nous constituons deux types de répertoires différents, mais tous deux fonctionnels pour la recherche. Le ReTeHNor est un répertoire «exact», exhaustif, qui n'admet plus d'enrichissement et recompositions (à moins de nouvelles découvertes, nouvelles recherches ou datations, attributions, etc.). Le second, l'Appendice, est un répertoire «inexact», non exhaustif et, contrairement au ReTeHNor, il n'est pas cohérent mais seulement homogène (c'est-à-dire qu'il contient des textes hagiographiques et pas d'autres types de sources). Il s'agit surtout d'un répertoire ouvert, ajusté au ReTeHNor, qui respecte au moins l'une des prémisses heuristiques du ReTeHNor, en enfreignant les autres. Il semble donc que le problème de l'Appendice reste ouvert: on pourrait dire également que son existence reste valide tant qu'elle est raisonnée et raisonnable par rapport au ReTeHNor. En conclusion de ces réflexions, qui confirment que, par corpus, on désigne un objet particulier, fluide et changeant au fil des évolutions

La Vita de l'abbé de Bourgueil a pour hypotexte BHN LVI/BHL 4032a, rédigé probablement à l'abbaye Saint-Mesmin de Micy vers le milieu du $\mathrm{X}^{\mathrm{e}}$ siècle: il est intégré à l'Appendice car il n'a pas été écrit en Normandie (Le Maно, 2006). Un autre cas est celui de la Vita Herluini (BHN L / BHL 3836) par Gilbert Crispin, moine au Bec pendant 25 ans puis abbé de Westminster (1085-1117) : ce texte nous permet de connaître la fondation exceptionnelle de l'abbaye hautnormande ainsi que la vie de Lanfranc avant son départ pour Cantorbéry; pourtant, il n'a pas été composé en Normandie mais à Westminster vers 1117-1118 (VAUGHN, 1981, p. 63). Cette Vita a d'ailleurs été réécrite dans la même abbaye du Bec au cours du XII ${ }^{\mathrm{e}}$ siècle (BHN 51/ BHL 3837 ).

36. Sur la nécessité de recourir à des éléments externes aux critères du corpus, voir par exemple Kossmann, 2014. 
techniques et des perspectives, il convient plutôt d'utiliser l'appellation de répertoire, conformément à l'étymologie du mot qui, en signifiant «trouver", garde en même temps la valeur de l'acte de la recherche et celle de l'agencement d'une structure dans laquelle on peut s'orienter facilement, par le recours aux humanités numériques.

\section{Bibliographie}

BAEDORF, Balthasar, Untersuchungen über Heiligenleben der westlichen Normandie, Bonn, Georgi, 1913.

BuIn, Jean-Baptiste-Nicolas, Vies des saints du diocèse de Sées et histoire de leur culte, Laigle, Montauzé, 1873.

Bozóky, Edina, La politique des reliques de Constantin à Saint Louis, Paris, Beauchesne, 2006.

Bouet, Pierre, Desbordes, Olivier (éd.), Chroniques latines du Mont Saint-Michel $\left(I X^{e}-X I I^{e}\right.$ siècle). Les manuscrits du Mont Saint-Michel: textes fondateurs, Caen, Presses universitaires de Caen, 2009.

Burdick, Anne, Drucker, Johanna, Lunenfeld, Peter, Presner, Todd, Schnapp, Jeffrey, Digital_Humanities, Cambridge MA, MIT Press, 2012.

BROwn, Peter, Le culte des saints. Son essor et sa fonction dans la chrétienté latine, Paris, Éditions du Cerf, 1984.

Chibnall, Marjorie, «The Merovingian monastery of St Evroul in the light of conflicting traditions", Studies in Church History, 8, 1972, p. 31-40.

Delamare, René, Le calendrier de l'église d'Évreux: étude liturgique et hagiographique, Paris, Picard, 1919.

De VRiendt, François, Trigalet, Michel, «Littérature hagiographique et bases de données. À propos de deux projets en cours à l'Université de Namur », Le Médiéviste et l'ordinateur, 34, 1997, p. 5-16 [consultable en ligne: http://lemo.irht. cnrs.fr/34/mo3403.htm].

Dolbeau, François, «La Vie latine de sainte Céronne, ermite en Normandie. Essai d'interprétation d'une légende ", Analecta Bollandiana, 104, 1986, p. 55-78.

Dolbeau, François, «Les travaux français sur l'hagiographie médiolatine (1968-1998)», Hagiographica, 6, 1999, p. 23-68.

Dolbeau, François, «De la "Bibliotheca hagiographica latina" à la "Biblioteca agiografica italiana". Quelques points de méthode», Hagiographica, 1, 1994, p. 350-359.

Dolbeau, François, Heinzelmann, Martin, Poulin, Joseph-Claude, «Les sources hagiographiques composées en Gaule avant l'an Mil (SHG), inventaire, examen critique», Francia, 15, 1987, p. 701-771.

FournéE, Jean, Le culte populaire et l'iconographie des saints en Normandie. Étude générale, Cahiers Léopold Delisle, $\mathrm{n}^{\circ}$ spécial, 1973.

Geary, Patrick, Le vol de reliques au Moyen Âge, Paris, Aubier, 1993.

Goullet, Monique, Écriture et réécriture hagiographiques. Essai sur les Vies des saints dans l'Occident médiéval (VIII'-XIII' siècles), Turnhout, Brepols, 2005. 
Goullet, Monique, «La laudatio sanctorum dans le haut Moyen Âge, entre vita et éloge» in Le discours d'éloge entre Antiquité et Moyen Âge, Lionel Mary et Michel Sot (dir.), Paris, Picard, 2001, p. 141-152.

Heinzelmann, Martin, Translationberichte und andere Quellen des Reliquienkultes, Turnhout, Brepols, 1979.

Hinkner, Monique, «La Vie métrique de saint Laumer (BHL 4735) copiée par Orderic Vital», Analecta Bollandiana, 124, 2006, p. 261-334.

Kahn Herrick, Samantha, Imagining the Sacred Past: Hagiography and Power in Early Normandy, Cambridge, Mass. et Londres, Harvard university Press, 2007.

Kossmann, Perrine, «Réflexion sur le corpus d'une thèse d'histoire ancienne intitulée Les lagides et l'Asie mineure", Annales de Janua, 2, 2014 [consultable en ligne: URL: http://annalesdejanua.edel.univ-poitiers.fr/index.php?id=679].

Le Hü̈rou, Armelle, Baudri de Bourgueil. Euvres en prose (textes hagiographiques), t. III, Paris, Les Belles Lettres, 2013.

Le Maho, Jacques, «Autour de la renaissance monastique du $\mathrm{X}^{\mathrm{e}}$ siècle en Normandie: les Vies des saints Aycadre et Hugues de Jumièges", in Livrets, collections et textes. Études sur la tradition hagiographique latine, Martin Heinzelmann (dir.), Ostfildern, Thorbecke (Beihefte der Francia, Bd. 63), 2006, p. 285-322.

Leonardi, Claudio, "Agiografia», in Lo spazio letterario del Medioevo, 1. Il Medioevo latino, t. II, Salerno Editore, Roma, 1993, p. 421-462.

Les saints dans la Normandie médiévale, Actes du colloque de Cerisy-la-Salle, 26-29 septembre 1996, Pierre Bouet et François Neveux (éd.), Caen, 2000.

Magnani, Eliana, Compte rendu de la journée d'études «Qu'est-ce qu'un corpus?», 2017, https://irht.hypotheses.org/3187.

MagnANi, Eliana, "Un corpus structuré et hétérogène de textes latins médiévaux (Bourgogne, $\mathrm{V}^{\mathrm{e}}-\mathrm{XV} \mathrm{e}^{\mathrm{e}}$ siècles) », Bulletin du CERCOR - Centre Européen de recherches sur les congrégations et ordres religieux, 41, 2017, p. 59-65.

MAyAfFre, Damon, "Le corpus réflexifs: entre architextualité et hypertextualité », Corpus, 1, 2002, p. 51-69 [consultable en ligne: http://journals.openedition.org/ corpus/11].

MAyAfFre, Damon, «Corpus et web-corpus. Réflexion sur la corporalité numérique», Cahiers de praxématique, 54-55, 2010, p. 233-248 [consultable en ligne: http:// journals.openedition.org/praxematique/1170].

Mesnel, Jean-Baptiste, Les saints du diocèse d'Évreux, Évreux, Hérissey, 1912-1918, 6 vol.

Musset, Lucien, "Observations sur le culte de sainte Marie-Madeleine en Normandie et notamment à Bayeux", Bulletin de la Société des antiquaires de Normandie, 56, 1961-1962, p. 667-670.

Musset, Lucien, «L'exode des reliques du diocèse de Sées au temps des invasions normandes ", Bulletin de la Société historique et archéologique de l'Orne, 88, 1970, p. 3-22.

Musset, Lucien, «De saint Victrice à saint Ouen: la christianisation de la province de Rouen d'après l'hagiographie», Revue d'histoire de l'Église de France, 62, 168, 1975, p. 141-152. 
Philippart, Guy, Hagiographies. Histoire internationale de la littérature hagiographique latine et vernaculaire en Occident des origines à 1550, Turnhout, Brepols (Corpus Christianorum, Hagiographies, 1), 1994, p. 10-17.

Philippart, Guy, «Le manuscrit hagiographique latin comme gisement documentaire. Un parcours dans les Analecta Bollandiana de 1960 à 1989 ", dans Manuscrits hagiographiques et travail des hagiographes, Martin Heinzelmann (éd.), Sigmaringen, Jan Thorbecke Verlag (Beihefte der Francia, 24), 1992, p. 17-48.

Pierazzo, Elena, Digital Scholarly Editing: Theories, Models and Methods, Farnham, Ashgate, 2015.

Pigeon, Émile-Aubert, Vies des saints du diocèse de Coutances et Avranches: avec des notions préliminaires et l'histoire des reliques de chaque saint, Avranches, Perrin, 1891.

Potтs, Cassandra, «When the saints go marching: Religious connections and the political culture of early Normandy", Anglo-Norman political culture and the twelfth-century renaissance: proceedings of the Borchard Conference on AngloNorman History, Charles Warren Hollister (éd.), 1995, p. 17-32.

Richard, Jean-Claude, Les «miracula» composés en Normandie aux XI et XII siècles, thèse de l'École des chartes, dact., Paris, 1975 (résumé in Positions des thèses de l'École nationale des chartes, 1975, p. 183-189).

Tognini-Bonelli, Elena, Corpus Linguistics at Work (Studies in Corpus Linguistics, 6), Amsterdam-Philadelphia, John Benjamins Publishing, 2001.

Tougard, Albert, Catalogue des saints du diocèse de Rouen, Rouen, Fleury, 1872.

TougarD, Albert, Essai sur l'hagiographie légendaire du diocèse de Rouen, Dieppe, Leprêtre, 1884.

Tougard, Albert, La Vie de saint Romain, Rouen, Rouen, L. Gy, 1899.

Tougard, Albert, Vie de saint Saêns, abbé au diocèse de Rouen, traduite pour la première fois d'après les livres d'office de l'abbaye, Paris, Dumont, 1890.

Trân-Duc, Lucile, Le culte des saints dans la Normandie médiévale (IXe-XII ${ }^{e}$ siècle). Enjeux de pouvoir dans les établissements bénédictins du diocèse de Rouen. Thèse de doctorat d'Histoire, Université de Caen Normandie, 2015, 2 vol.

Treffort, Cécile, «Le corpus du chercheur, une quête de l'impossible? Quelques considérations introductives", Le corpus. Son contour, ses limites et sa cohérence, Annales de Janua, 2, 2014. [En ligne] http://annalesdejanua.edel.univ-poitiers.fr/ index.php?id=725.

Vaughn, Sally N., The Abbey of Bec and the Anglo-Norman State, 1034-1136, Woodbridge, Boydell Press, 1981.

Verrando, Giovanni, Nino, «Per una nuova "Bibliotheca Hagiographica Latina" compilata sui manoscritti di origine italiana", Hagiographica, 2, 1995, p. 277-308.

WALKER, Lorna E. M., «Hamo of Savigny and his companions: failed saints?», Journal of Medieval History, 30, 2004, p. 45-60. 